\title{
Altered Functional Connectivity of the Insula and Nucleus Accumbens in Internet Gaming Disorder: A Resting State fMRI Study
}

\author{
Chiao-Yun Chen ${ }^{a, c}$ Ju-Yu Yen ${ }^{b, d, e}$ Peng-Wei Wang ${ }^{b, d}$ Gin-Chung Liu ${ }^{a, c}$ \\ Cheng-Fang Yen ${ }^{b, d}$ Chih-Hung Ko ${ }^{b, d}, f$ \\ ${ }^{a}$ Department of Medical Imaging, ${ }^{b}$ Department of Psychiatry, Kaohsiung Medical University Hospital, ${ }^{c}$ Department \\ of Radiology, ${ }^{\mathrm{d}}$ Department of Psychiatry, Faculty of Medicine, College of Medicine, e Department of Psychiatry, \\ Kaohsiung Municipal Ta-Tung Hospital, and f Department of Psychiatry, Kaohsiung Municipal Hsiao-Kang Hospital, \\ Kaohsiung Medical University, Kaohsiung, Taiwan
}

\section{Key Words}

Internet gaming disorder · Functional connectivity - Insula .

Nucleus accumbens $\cdot$ Frontal lobe $\cdot$ Impulsivity

\begin{abstract}
Aims: A possible addiction mechanism has been represented by altered functional connectivity $(\mathrm{FC})$ in the resting state. The aim of this study was to evaluate the FCs of the insula and nucleus accumbens among subjects with Internet gaming disorder (IGD). Methods: We recruited 30 males with IGD and 30 controls and evaluated their FC using functional magnetic imaging scanning under resting, a state with relaxation, closed eyes, with inducement to think of nothing systematically, become motionless, and instructed not to fall asleep. Results: Subjects with IGD had a lower FC with the left insula over the left dorsolateral prefrontal cortex (DLPFC) and orbital frontal lobe and a higher FC with the insula with the contralateral insula than controls. The inter-hemispheric insula connectivity positively correlated with impulsivity. Further, they had lower FC with the left nucleus accumbens over the left DLPFC and with the right nucleus accumbens over the left DLPFC, and insula and a higher FC with that over the right precuneus. Conclusion: The elevated inter-hemispheric insula FC is found to be associated with impulsivity
\end{abstract}

and might explain why it is involved in IGD. The attenuated frontostriatal suggests that the emotion-driven gaming urge through nucleus accumbens could not be well regulated by the frontal lobe of subjects with IGD.

(C) 2016 S. Karger AG, Base

\section{Introduction}

\section{Internet Gaming Disorder}

The Internet is a facility that is available 24 hours a day; a loss of control of Internet use may result in negative psychosocial consequences [1]. The dys-control Internet use is labeled as Internet addiction [2]. Internet gaming disorder (IGD) is one of the more popular subtypes (57.5\%) of Internet addiction [3]. As for its significant public importance, the DSM 5 recruited IGD in Section III [4] and suggested that adequate evidence was necessary to support it as an independent disorder. Subjects with IGD have been reported to play online gaming for $5.87 \mathrm{~h} /$ day on weekdays and $8.51 \mathrm{~h} /$ day at weekends [5]. The long-term gaming experience might make a difference in brain function [6]. On the other hand, the alteration in brain functioning might represent characteristics such as impulsivity [7] that could predict IGD.

\section{KARGER}

E-Mail karger@karger.com

www.karger.com/ear
(C) 2016 S. Karger AG, Basel

$1022-6877 / 16 / 0224-0192 \$ 39.50 / 0$
Chih-Hung Ko

Department of Psychiatry

Faculty of Medicine, College of Medicine, Kaohsiung Medical University

100 Tzyou 1st Rd. Kaohsiung City 807 (Taiwan)

E-Mail cyberko@seed.net.tw 
The Functional Connectivity of IGD

There are behavioral similarities, such as impulsivity, of IGD to gambling disorder or substance use disorder [4, 8]. Evaluation of the resting-state functional connectivity (FC) provides a way to characterize distributed circuit abnormalities in neuropsychiatric illnesses, such as addictive disorder [9]. The default network resting-state connection of 15 subjects with IGD has been evaluated by regional homogeneity ( $\mathrm{ReHo}$ ). It demonstrated enhanced $\mathrm{ReHo}$ in the brainstem, inferior parietal lobule, left posterior cerebellum, and left middle frontal gyrus [10]. Another study showed higher ReHo over the cerebellum, brainstem, and multiple brain regions in 19 subjects of IGD [11]. Enhanced visual motor coordination and impaired visual and auditory function had been suggested in IGD based on the result of ReHo [10]. Further, altered default network resting-state FC to the posterior cingulate has been found in 17 adolescents with IGD [12]. These results demonstrate inconsistent results of FC in IGD.

All previous studies diagnosed the subjects based on a self-reported questionnaire. Psychiatric interviewing could contribute to a more precious diagnosis. Moreover, the sample size of these studies is smaller than the optimal sample size suggested in previous reviews [13]. Lastly, these previous studies investigated a whole brain FC. Evaluation of the FC to specific brain regions should be examined to shed light on the mechanisms of IGD.

\section{The Insula and Addiction}

The insula has a role in the salience network and plays a causal role in the initiation, maintenance, and adjustment of attention control. It helps individuals pay attention to the current most salient stimuli [14]. The frontoinsular cortex plays a critical role in activating the central executive network, such as the dorsolateral prefrontal cortex (DLPFC) [13]. Thus, the connectivity within the insula, inferior frontal lobe, and DLPFC has been suggested as involving a cognitive control network [15]. Further, the inter-network connectivity involving the insula has been reported to be weaker in cocaine users and related to their impulsivity [16]. The insular-striatal connection also mediates the association between impulsivity and cocaine relapse [17]. Moreover, inter-hemispheric insular FC is associated with addictive disorder [18]. These results support the fact that FC of insula could be involved in the addiction process.

Subjects with IGD have been reported to have decreased cortex thickness [19], lower white matter density [20], and impaired error-process activation over insula
[21]. Based on the previous studies, we hypothesized that FC between the insula, inferior frontal lobe, and DLPFC is associated with IGD.

\section{The Nucleus Accumbens and Addiction}

The nucleus accumbens has a well-established role in mediating the rewarding effects of drugs abuse and natural rewards such as food and sexual behavior [22]. Further study demonstrated that the FC of nucleus accumbens, particularly its interaction with the prefrontal lobe, was associated with impulsivity [23]. Both exposures to methylphenidate or alcohol cues could decrease its FC to the frontal lobe [24, 25]. Further, the disrupted FC of nucleus accumbens and DLPFC has been reported to be associated with substance use disorder [26]. These results indicate that addiction might be associated with altered connectivity between the nucleus accumbens that process reward and DLPFC that govern the cognitive-behavioral control.

The nucleus accumbens has been reported to be activated in cue-induced gaming urge [27]. Recent research suggested that the higher activation of the nucleus accumbens at rest may indicate constant activation in the context of a diminished prefrontal control and association with severity of Internet addiction [28]. However, FC of the nucleus accumbens and its role in impulsivity have not been evaluated in subjects with IGD. Thus, we hypothesized that its FC to the prefrontal lobe decreased among subjects with IGD.

\section{Impulsivity and Addiction}

Impulsivity includes deficits in attention, lack of reflection, and insensitivity to consequences. It is one of the most important factors contributing to IGD [29]. As the loss of control in online gaming is one essential criterion of IGD [4], high impulsivity could make subjects yield to the rewarding effect of gaming and contribute to the vulnerability of IGD. Further, the impulsivity has been reported to correlate with FC of the insula and nucleus accumbens $[17,23]$, particularly to the frontal lobe [15]. Thus, we hypothesized that impulsivity may influence the vulnerability of IGD by impacting its neurobiological underpinnings in FC of the insula and nucleus accumbens.

Thus, this study aimed at evaluating the alteration in the FCs of the insula and nucleus accumbens among adults with IGD in comparison to controls. According to previous studies, we hypothesized that the FC of the insula over the prefrontal lobe and contralateral insula and that of the nucleus accumbens over the prefrontal lobe and insula were altered among adults with IGD. Further, FCs of these 
brain regions play essential roles in impulsivity [17, 23], which is one possible mechanism of IGD. Thus, we evaluated the correlation between the FCs of the insula and nucleus accumbens and impulsivity in this study.

\section{Methods}

\section{Participants}

Male right-handed participants were recruited by placing an advertisement. All subjects in the IGD group were interviewed by a psychiatrist to confirm a diagnosis of IGD for more than 2 years according to the diagnostic criteria for Internet addiction (DCIA) [30]. They were currently addicted to online gaming and spent an average of 4 or more hours per day on weekdays and an average of 8 or more hours per day on weekends to play online games. In the control group, psychiatry-based interviews and reviews of history were performed to confirm that none had ever fulfilled the DCIA. The exclusion criteria were assessed by a psychiatrist and included the current use of psychotropic medication and any history of substance use disorder (excluding nicotine dependence), major depressive episode, bipolar I disorder, psychotic disorder, neurological illness or injury, mental retardation, or poor tolerance of magnetic resonance imaging. All subjects gave written informed consent after receiving a detailed explanation of the study. The study was approved by the Kaohsiung Medical University Institutional Review Board.

\section{Image Acquisition}

The fMRI experiments were performed with a 3-tesla General Electric MR scanner (Signa VH/i, software: version 4.0). The magnetic resonance sequence for functional imaging was a gradientrecalled echo planar imaging $(\mathrm{EPI})$ sequence $(64 \times 64$ matrix; 24 $\mathrm{cm}$ field of view, echo time $=35 \mathrm{~ms}$; repetition time $=2 \mathrm{~s} ; 4-\mathrm{mm}$ thick slices with a 0 -mm gap). Twenty-eight image planes were collected parallel to the anterior commissure and posterior commissure line with the aid of sagittal localizer images. The scanning time of EPI was $620 \mathrm{~s}$ in total. T1-weighted anatomical images were obtained using FSPGR (Spoiled Gradient Echo) sequence $(256 \times$ 256 matrix; $24-\mathrm{cm}$ field of view, echo time $=2.8-12.0 \mathrm{~ms}$; repetition time $=6.6 \mathrm{~ms} ; 1-\mathrm{mm}$-thick slices with a $0.47-\mathrm{mm}$ gap) before fMRI scanning to enable anatomical registration. The resting state was defined as performing no specific cognitive task during fMRI scanning [31]. Subjects were required to relax, close their eyes, think of nothing systematically, remain motionless, and not fall asleep. After scanning, every subject was questioned as to their waking state during scanning, and none reported of having fallen asleep.

Process

All subjects completed the Chen Internet Addiction Scale (CIAS) [32] and the Barratt Impulsivity Scale 11 (BIS-11) [33, 34] before scanning. The CIAS, QGU-B, and BIS-11 scores indicated the severity of Internet addiction and the severity of impulsivity respectively.

The first 5 volumes of the functional time series were discarded; 305 volumes were preprocessed using the SPM8 package (Wellcome Department of Cognitive Neurology, London, UK). First, realign for the head motion was performed. The excessive motion was defined as more than $2 \mathrm{~mm}$ of translation or more than 2 degrees of rotation in any direction. Data of 2 IGD subjects and 2 healthy subjects were excluded from further analysis according to these criteria. Then we coregistered the high-resolution individual T1-weighted images to the individual mean EPI. The realigned datasets were then normalized to Montreal Neurological Institute space and resliced in a $3 \times 3 \times 3 \mathrm{~mm}^{3}$ voxel size. A 4 -mm fullwidth-half-maximum Gaussian kernel was used to smooth the data.

Further preprocessing was performed using the Resting-State fMRI Data Analysis Toolkit (REST, by Song et al: http://restingfmri.sourceforge.net), including removal of the linear trend and temporal band-pass filtering $(0.01-0.08 \mathrm{~Hz})$ to reduce low-frequency drift as well as high-frequency respiratory and cardiac noise.

\section{Data Analysis}

Anatomical regions of interest (ROIs) were created from the AAL library as found in WFU Pickatlas version 2.4 [35]. Insula was selected because of their essential roles in the salience network [36]. The seeds of the nucleus accumbens were located at $X= \pm 9$, $\mathrm{Y}=9, \mathrm{Z}=-8$, with $3.5-\mathrm{mm}$ radial spheres (sampling approximately 25 voxels in $2 \mathrm{~mm}$ of isotropic resolution) according to previous studies $[13,37]$.

Calculation of functional connection was carried out using REST. For each ROI, a seed reference time course was obtained by averaging the time series of all voxels in the ROI. Then, Pearson's correlation analysis was performed between the seed reference time course and the time series from the whole brain in a voxelwise way, with the global signal, WM signal, CSF signal and the 6 head-motion parameters as nuisance covariates. Finally, the resultant correlation coefficients were transformed into z-scores using Fisher's transformation for better satisfaction of normality.

Analysis of imaging data was carried out using SPM8. First, random-effect one-sample $t$ tests were conducted on the individual Z-maps of the IGD or control group separately, producing 4 t-maps corresponding to the FC patterns of the left and right insula, or nucleus accumbens ROIs in the IGD and control groups. The differences on t-maps between the IGD and control groups were determined with a threshold of $\mathrm{p}<0.001$ uncorrected at the voxel level in combination with a level of $\mathrm{p}<0.05$ with family-wise error (FWE) small volume correction at the cluster level. According to the previous results, the FCs of insula and nucleus accumbens over the orbital frontal lobe (OFL) [38] and DLPFC $[15,26]$ had been reported to be associated with addiction. The OFL, DLPFC, and insula were selected as small volumes in this analysis. The correlation between the score of BIS-11 and FCs of insula and nucleus accumbens among all subjects were evaluated by multiple regression in SPM. The significant correlations were determined with a threshold of $\mathrm{p}<0.001$ uncorrected at the voxel level in combination with a level of $\mathrm{p}<0.05$ with FWE at the cluster level.

\section{Results}

A total of 28 male subjects with IGD and 28 male controls were recruited for the final analysis. The demographic data presented in table 1 show that the IGD and 
Table 1. Differences in age, educational level, the severity of the IGD ${ }^{\mathrm{a}}$ and impulsivity between subjects with IGD and controls

\begin{tabular}{lllc}
\hline Variable & $\begin{array}{l}\text { IGD } \\
(\text { mean } \pm \text { SD) }\end{array}$ & $\begin{array}{l}\text { Control }^{\mathrm{b}} \text { group } \\
(\text { mean } \pm \text { SD) }\end{array}$ & $\mathrm{t}$ \\
\hline Age, years & $23.64 \pm 2.54$ & $24.14 \pm 2.53$ & -0.73 \\
Educational level, years & $15.86 \pm 1.35$ & $16.50 \pm 1.40$ & -1.74 \\
IGD severityc $_{\text {Impulsivity }}$ & $83.14 \pm 10.26$ & $34.39 \pm 11.11$ & $17.60^{*}$ \\
\hline
\end{tabular}

${ }^{a}$ IGD: subjects with IGD for more than 2 years.

b Control: subjects with no history of IGD.

${ }^{c}$ IGD severity: score of the CIAS.

d Impulsivity: score of BIS-11.

$* \mathrm{p}<0.001$.

control groups did not differ significantly in terms of age or educational levels. The IGD group had significantly higher CIAS, and BIS-11 scores than the control group.

\section{Between-Group Differences in the FC Pattern of the} Insula Shown Table 2 and Figure 1

The IGD group had significantly lower FC with the left insula over the left DLPFC, and OFL (fig. 1a) than the control group. However, no significant lower FC with right insula was noted within hypothesized regions.

On the other hand, the IGD group had a significantly higher FC with the left insula over the right insula (fig. 1c) and a higher FC with the right insula over the left insula (fig. 1d) than the control group.

\section{Between-Group Differences in the FC Pattern of the} Nucleus Accumbens Shown in Table 2 and Figure 2

The IGD group had a significantly lower FC with the right nucleus accumbens over the left DLPFC and right insula (fig. 2b) than the control group. Further, the IGD group exhibited a lower FC with the left nucleus accumbens over the left DLPFC than the control group.

The IGD group had a significantly higher FC with the left or right nucleus accumbens over the right precuneus (fig. $2 \mathrm{c}$ and d) than the control group. Although the precuneus was not included in the hypothesized region in small volume correction, it was significant in whole-brain FEW correction at the cluster level.

\section{The Correlation between Impulsivity and FCs of}

Insula and Nucleus Accumbens in Figure 1e and $f$

The regression analysis demonstrated that impulsivity was positively correlated with FC of the left insula over the right insula $(X=39, Y=-12, Z=-3 ; 156$ voxels; $Z=4.5$; $\mathrm{p}<0.001$ in small volume FEW correction; fig. 1e) and with FC of the right insula over the left insula $(X=-42$, $\mathrm{Y}=6, \mathrm{Z}=6 ; 1,112$ voxels; $\mathrm{Z}=4.46 ; \mathrm{p}<0.001$ in small volume FEW correction; fig. 1f). This result demonstrated the positive correlation between impulsivity and inter-hemisphere insula connectivity. However, there was no significant correlation found in FCs of the nucleus accumbens.

\section{Discussion}

\section{The Functional Connection of the Insula}

The insula has been suggested to be involved in the following processes: (1) incentive motivational processes that drive motivation for substance; (2) control processes that inhibit addictive behavior; and (3) interoceptive processes that represent substance-related bodily states [39]. The inter-hemispheric insula FC was reported to associate with substance abuse among psychiatric patients [18]. This study demonstrated that subjects with IGD had higher inter-hemispheric insula FC than the control group. It would suggest that the inter-hemispheric insula FC was involved not only in substance use disorder but also in IGD. Playing online games could bring about a hedonistic experience with a physical hyper-arousal reaction, particularly in first-person shot games [40]. As the insula plays a central role in conscious interoception and emotional feeling, it represented the interoceptive stimuli during the hedonic experience and recalled this experience from memory [39]. Thus, the repeated online gaming and excessive use every day might enhance the inter-hemispheric insula connection among subjects with IGD. However, the claim should be evaluated in a 
Table 2. Differences in FC with the insula and nucleus accumbens between subjects with IGD and controls

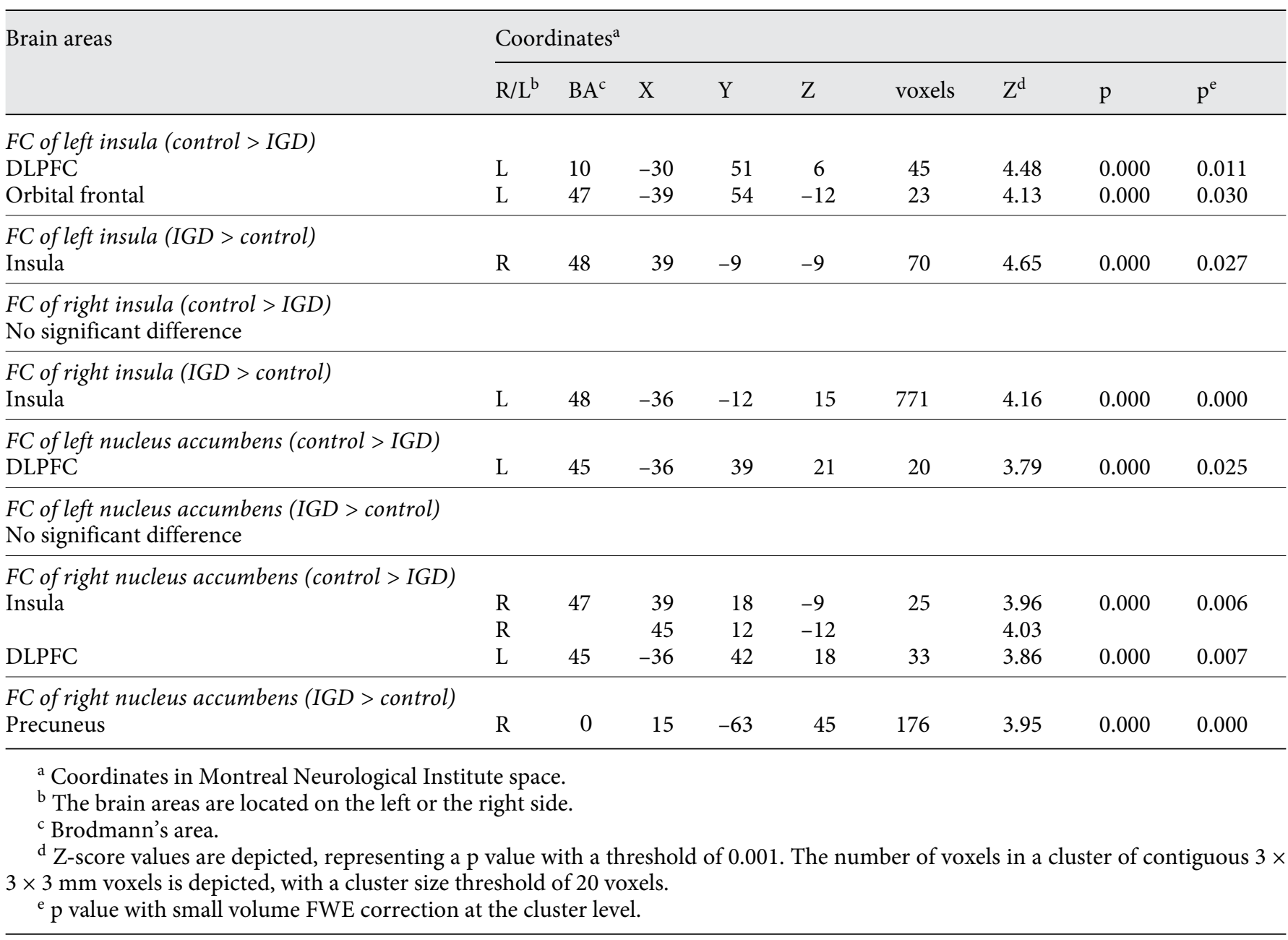

future study for the connecting state when exposure to gaming cues.

Our study also demonstrated that inter-hemispheric insula connectivity was positively associated with impulsivity. It suggested that the higher the inter-hemispheric insula connection, the higher the impulsivity. Insula has been suggested to involve the appetite motivation system [39] and represent a rewarding experience. The stronger interhemispheric connection could provide an intense interoception and emotional feeling and drive higher motivation under immediate reward. It could contribute to vulnerability to a lower, but immediate reward over a delayed, but more valuable outcome, one dimension of impulsivity [41]. Its correlation to impulsivity might also explain why it was repeatedly reported to be associated with substance and behavioral addiction. However, as the connectivity was evaluated in the resting state, the role of inter-hemi- spheric insula connectivity in impulsivity should be evaluated in further FC study under cognitive control task.

On the other hand, insula also involves a control system when connecting to the anterior cingulate or prefrontal lobe $[15,39]$. The frontoinsular cortex plays a critical role in activating the central executive network [42]. The insula-DLPFC connectivity under the stop-signal task has been reported to negatively associate with impulsivity [43]. In this study, subjects with IGD had lower FC with the left insula over the left DLPFC and OFL. A recent study demonstrated that subjects with IGD had decreased activation of error processing over insula and needed to activate higher OFL function to maintain their response inhibition function [21]. Although the impaired frontalinsula connectivity was noted among subjects with IGD in this study, the frontal-insula connectivity was not correlated with impulsivity. Evaluation of the frontal-insula 
Fig. 1. The brain areas with altered FC to insula among subjects with Internet gaming group in comparison to control group and the brain correlates of impulsivity in FC to insula. a The brain areas with lower FC to left insula among IGD group than those among control group ( $\mathrm{p}<0.05$ in FWE small volume correction in cluster level). b The brain areas higher FC to left insula among IGD group than those among control group ( $p<0.05$ in FWE small volume correction in cluster level). $c$ The brain areas with lower FC to right insula among IGD group than those among control group ( $p<0.05$ in FWE small volume correction in cluster level). d The brain areas higher FC to right insula among IGD group than those among control group ( $\mathrm{p}<0.05$ in FWE small volume correction in cluster level). e The brain correlates of impulsivity in FC of left insula ( $\mathrm{p}<0.05$ in FWE correction in cluster level). $\mathbf{f}$ The brain correlates of impulsivity in FC of right insula ( $\mathrm{p}<0.05$ in FWE correction in cluster level).

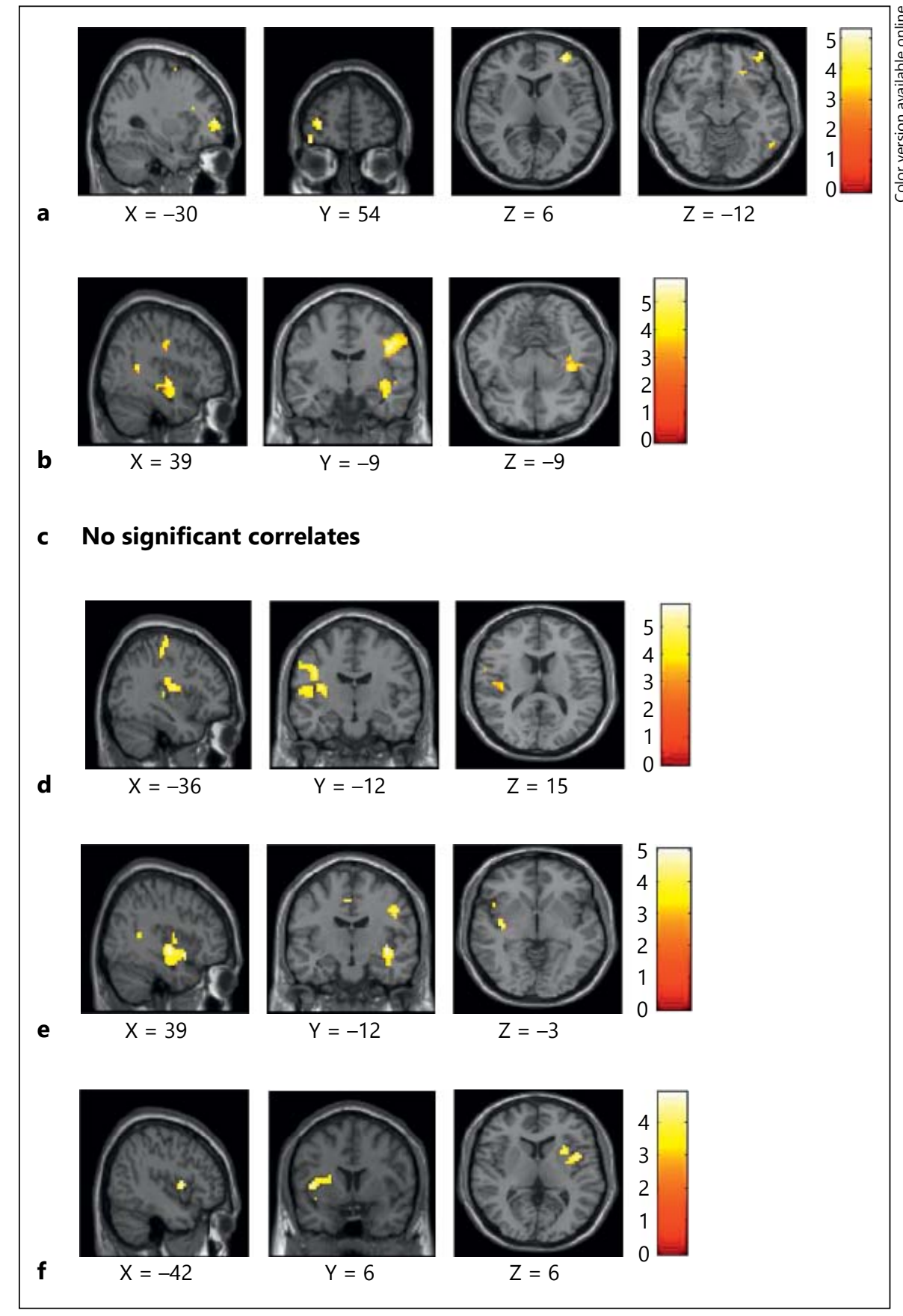

connectivity under tasks of specific functions should be considered to validate its role in the addiction process of IGD.

The Functional Connection of the Nucleus Accumbens

The nucleus accumbens linked motivation to action by integrating information from limbic structures and the prefrontal cortex [44]. The DLPFC are involved in inhibitory control, emotion regulation, and decisionmaking [45]. The disrupted frontal-striatal connectivity has been found to be associated with substance use disorder [26]. It suggests that addiction could be associated with abnormal interactions between the rewarding process and cognitive control. The elevated limbic arousal response in nucleus accumbens combined with a depressed cognitive control function in DLPFC might re- 


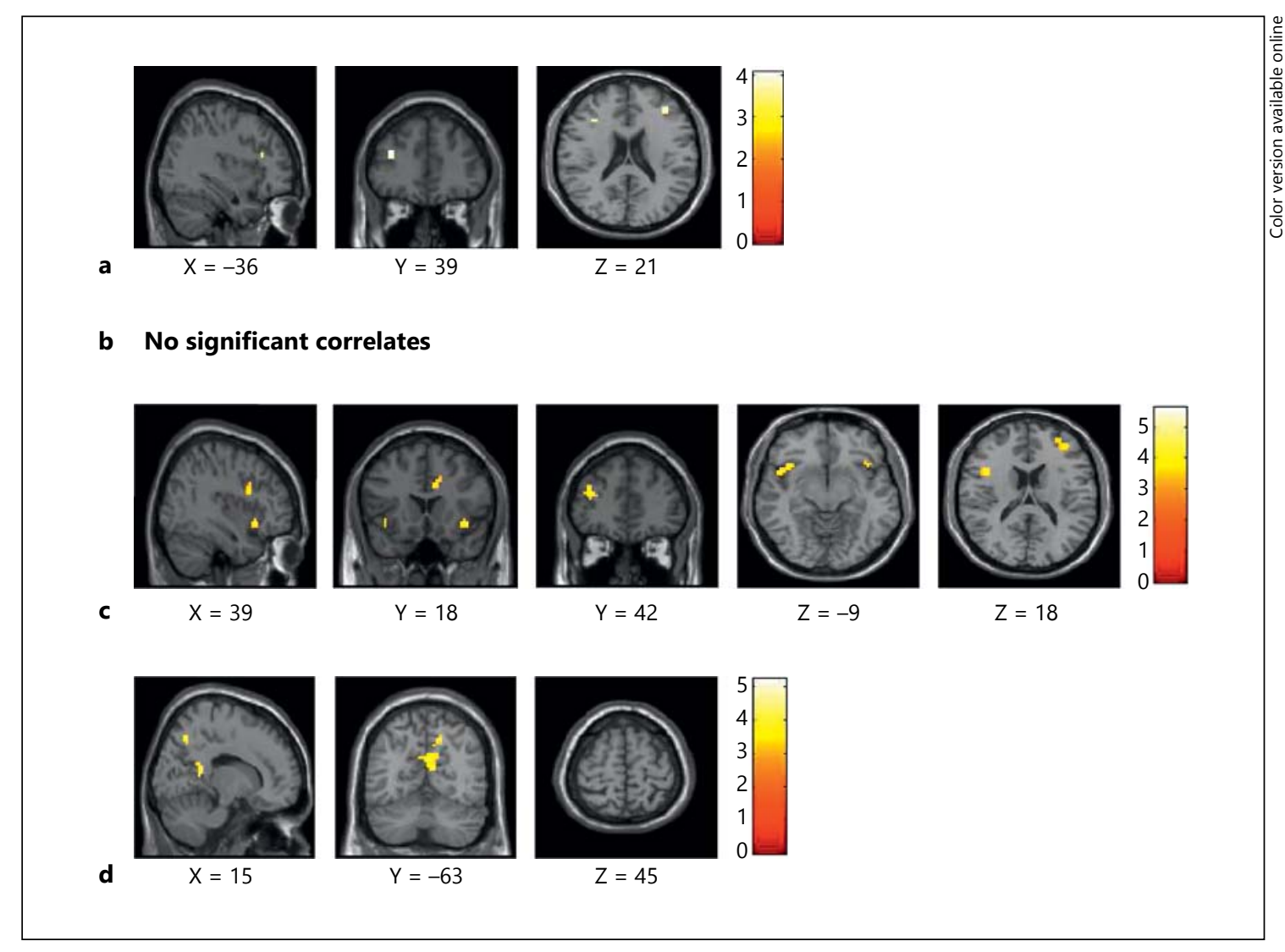

Fig. 2. The brain areas with altered FC to nucleus accumbens among subjects with Internet gaming group in comparison to control group. a The brain areas with lower FC to left nucleus accumbens among the IGD group than those among the control group ( $p<0.05$ in FWE small volume correction in cluster level). $\mathbf{b}$ The brain areas with higher FC to left nucleus accumbens among the IGD group than those among the control group $(\mathrm{p}<0.05$ in FWE small volume correction in cluster level). No significant correlates. c The brain areas with lower FC to right nucleus accumbens among the IGD group than those among the control group $(\mathrm{p}<0.05$ in FWE small volume correction in cluster level). $\mathbf{d}$ The brain areas with higher FC to right nucleus accumbens among the IGD group than those among the control group $(\mathrm{p}<0.05$ in FWE small volume correction in cluster level). sult in uncontrolled addictive behavior under rewarding cue [46]. This study revealed that subjects with IGD had significantly lower FC with the right nucleus accumbens over the left DLPFC and right insula. They also exhibited lower FC with the right nucleus accumbens over the left DLPFC. The nucleus accumbens has been found to activate for cue-induced gaming urge in IGD [27]. Our result supported the disrupted frontal-striatal connectivity involved in the mechanism of IGD. However, further study to evaluate the frontal-striatal connectivity under gaming urge is necessary to understand its role in IGD.

The nucleus accumbens-precuneus connectivity has been found to be associated with a sensation-seeking behavior and alcohol use among the youth with a family history of alcoholism [47]. The precuneus is involved in not only cue-induced alcohol craving [48] but also online gaming urges [49]. This study demonstrated a higher nucleus accumbens-precuneus connectivity of IGD and has suggested the role of this connectivity in mechanism of IGD. However, how it plays a role in IGD should be evaluated in the future.

\section{Clinical Implications}

Our result demonstrated that the insula, the center of salience network [50], and the nucleus accumbens, the center of motivation [44], had lower FC to frontal lobe among subjects with IGD. Besides, the inter-hemispheric insula connectivity was stronger in IGD. Further well-designed studies are necessary to evaluate the role of these altered FCs in IGD. To evaluate these FCs among subjects' remission from IGD might 
provide insight as to whether this alteration is a state marker or a vulnerability characteristic of IGD. On the other hand, an intervention such as transcranial magnetic modulation $[46,51]$, which could moderate the frontal-striatal circuit, should be considered to be one potential treatment of IGD and is deserving of further research.

\section{Limitations}

Several limitations of this study should be noted. First, only males were included in the study. Second, as cases comorbid with substance abuse and other major psychiatric disorders were excluded in this study, there is a limitation in generalizing the results to subjects with IGD comorbidity with other substance use disorders or major psychiatric disorders. Third, the interpretations of the results of FC were based on a literature review. The functions of FC in the insula and nucleus accumbens were not proven in this study. Lastly, the causal relationships between IGD and the findings of FC could not be confirmed in this cross-sectional study.

\section{Conclusion}

Despite the above limitations, this study demonstrates the elevated inter-hemispheric insula FC and attenuated nucleus accumbens-DLPFC connectivity among subjects with IGD. The inter-hemispheric insula FC was positively correlated with impulsivity. These results might suggest that elevated inter-hemispheric insula FC might be involved in impulsivity and contribute to the mechanism of IGD. The disrupted frontal-striatal connectivity had its essential role in IGD just as its role in substance use disorder. Thus, more attention should be paid to the disconnectivity between the insula, nucleus accumbens and frontal lobe and impulsivity in the treatment of subjects with IGD.

\section{Acknowledgments}

This study was supported by grants from the National Science Council (MOST103-2314-B-037-009), Kaohsiung Municipal Hsiao-Kang Hospital (KMHK-102-006), and the Kaohsiung Medical University Hospital (KMUH102-2R57).

\section{References}

1 Young KS, Case CJ: Internet abuse in the workplace: new trends in risk management. Cyberpsychol Behav 2004;7:105-111.

2 Yen CF, Yen JY, Ko CH: Internet addiction: ongoing research in Asia. World Psychiatry 2010;9:97.

3 Kishi T, Tsunoka T, Ikeda M, Kawashima K, Okochi T, Kitajima T, Kinoshita Y, Okumura $\mathrm{T}$, Yamanouchi Y, Inada T, Ozaki N, Iwata N: Serotonin 1A receptor gene and major depressive disorder: an association study and metaanalysis. J Hum Genet 2009;54:629-633.

4 American Psychiatric Association: Diagnostic and Statistical Manual of Mental Disorders, ed 5. Arlington, American Psychiatric Association, 2013.

5 Ko CH, Yen JY, Chen SH, Wang PW, Chen CS, Yen CF: Evaluation of the diagnostic criteria of Internet gaming disorder in the DSM5 among young adults in Taiwan. J Psychiatr Res 2014;53:103-110.

6 Bavelier D, Green CS, Pouget A, Schrater P: Brain plasticity through the life span: learning to learn and action video games. Annu Rev Neurosci 2012;35:391-416.

7 Gentile DA, Choo H, Liau A, Sim T, Li D, Fung D, Khoo A: Pathological video game use among youths: a two-year longitudinal study. Pediatrics 2011;127:e319-e329.

8 Ko CH, Yen JY, Chen CC, Chen SH, Yen CF: Proposed diagnostic criteria of Internet addiction for adolescents. J Nerv Ment Dis 2005; 193:728-733.
9 Sutherland MT, McHugh MJ, Pariyadath V, Stein EA: Resting state functional connectivity in addiction: lessons learned and a road ahead. Neuroimage 2012;62:2281-2295.

10 Dong G, Huang J, Du X: Alterations in regional homogeneity of resting-state brain activity in Internet gaming addicts. Behav Brain Funct 2012;8:41.

11 Liu J, Gao XP, Osunde I, Li X, Zhou SK, Zheng $\mathrm{HR}, \mathrm{Li}$ LJ: Increased regional homogeneity in Internet addiction disorder: a resting state functional magnetic resonance imaging study. Chin Med J (Engl) 2010;123:1904-1908.

12 Ding WN, Sun JH, Sun YW, Zhou Y, Li L, Xu JR, Du YS: Altered default network restingstate functional connectivity in adolescents with Internet gaming addiction. PLoS One 2013;8:e59902.

13 Harrison BJ, Soriano-Mas C, Pujol J, Ortiz H, López-Solà $\mathrm{M}$, Hernández-Ribas R, Deus J, Alonso P, Yücel M, Pantelis C, Menchon JM, Cardoner N: Altered corticostriatal functional connectivity in obsessive-compulsive disorder. Arch Gen Psychiatry 2009;66:11891200 .

14 Menon V, Uddin LQ: Saliency, switching, attention and control: a network model of insula function. Brain Struct Funct 2010;214: 655-667.

15 Sundermann B, Pfleiderer B: Functional connectivity profile of the human inferior frontal junction: involvement in a cognitive control network. BMC Neurosci 2012;13:119.
16 Wisner KM, Patzelt EH, Lim KO, MacDonald AW 3rd: An intrinsic connectivity network approach to insula-derived dysfunctions among cocaine users. Am J Drug Alcohol Abuse 2013;39:403-413.

17 McHugh MJ, Demers CH, Braud J, Briggs R Adinoff B, Stein EA: Striatal-insula circuits in cocaine addiction: implications for impulsivity and relapse risk. Am J Drug Alcohol Abuse 2013;39:424-432.

18 Viswanath $H$, Velasquez KM, Savjani R, Molfese DL, Curtis K, Molfese PJ, Eagleman DM, Baldwin PR, Frueh BC, Fowler JC, Salas $\mathrm{R}$ : Interhemispheric insular and inferior frontal connectivity are associated with substance abuse in a psychiatric population. Neuropharmacology 2015;92:63-68.

19 Yuan K, Cheng P, Dong T, Bi Y, Xing L, Yu D, Zhao L, Dong M, von Deneen KM, Liu Y, Qin W, Tian J: Cortical thickness abnormalities in late adolescence with online gaming addiction. PLoS One 2013;8:e53055.

20 Lin X, Dong G, Wang Q, Du X: Abnormal gray matter and white matter volume in 'Internet gaming addicts'. Addict Behav 2015;40: 137-143.

$21 \mathrm{Ko} \mathrm{CH}$, Hsieh TJ, Chen CY, Yen CF, Chen CS, Yen JY, Wang PW, Liu GC: Altered brain activation during response inhibition and error processing in subjects with Internet gaming disorder: a functional magnetic imaging study. Eur Arch Psychiatry Clin Neurosci 2014;264:661-672. 
22 Koob GF, Volkow ND: Neurocircuitry of addiction. Neuropsychopharmacology 2010;35: 217-238.

23 Behan B, Stone A, Garavan H: Right prefrontal and ventral striatum interactions underlying impulsive choice and impulsive responding. Hum Brain Mapp 2015;36:187-198.

24 Ramaekers JG, Evers EA, Theunissen EL, Kuypers KP, Goulas A, Stiers P: Methylphenidate reduces functional connectivity of nucleus accumbens in brain reward circuit. Psychopharmacology (Berl) 2013;229:219-226.

25 Ray LA, Courtney KE, Hutchison KE, Mackillop J, Galvan A, Ghahremani DG: Initial evidence that OPRM1 genotype moderates ventral and dorsal striatum functional connectivity during alcohol cues. Alcohol Clin Exp Res 2014;38:78-89.

26 Motzkin JC, Baskin-Sommers A, Newman JP, Kiehl KA, Koenigs M: Neural correlates of substance abuse: reduced functional connectivity between areas underlying reward and cognitive control. Hum Brain Mapp 2014;35: 4282-4292.

27 Ko CH, Liu GC, Hsiao S, Yen JY, Yang MJ, Lin WC, Yen CF, Chen CS: Brain activities associated with gaming urge of online gaming addiction. J Psychiatr Res 2009;43:739-747.

28 Kühn S, Gallinat J: Brains online: structural and functional correlates of habitual Internet use. Addict Biol 2015;20:415-422.

29 Ding WN, Sun JH, Sun YW, Chen X, Zhou Y, Zhuang ZG, Li L, Zhang Y, Xu JR, Du YS: Trait impulsivity and impaired prefrontal impulse inhibition function in adolescents with Internet gaming addiction revealed by a Go/No-Go fMRI study. Behav Brain Funct 2014;10:20.

$30 \mathrm{Ko} \mathrm{CH}$, Yen JY, Chen SH, Yang MJ, Lin HC, Yen CF: Proposed diagnostic criteria and the screening and diagnosing tool of Internet addiction in college students. Compr Psychiatry 2009;50:378-384.

31 Biswal B, Yetkin FZ, Haughton VM, Hyde JS: Functional connectivity in the motor cortex of resting human brain using echo-planar MRI. Magn Reson Med 1995;34:537-541.
32 Chen SH, Weng LJ, Su YJ, Wu HM, Yang PF: Development of Chinese Internet addiction scale and its psychometric study. Chin J Psychol 2003;45:279-294.

33 Li CS, Chen SH: Obsessive-compulsiveness and impulsivity in a non-clinical population of adolescent males and females. Psychiatry Res 2007;149:129-138.

34 Patton JH, Stanford MS, Barratt ES: Factor structure of the Barratt impulsiveness scale. J Clin Psychol 1995;51:768-774.

35 Tzourio-Mazoyer N, Landeau B, Papathanassiou D, Crivello F, Etard O, Delcroix N, Mazoyer B, Joliot M: Automated anatomical labeling of activations in SPM using a macroscopic anatomical parcellation of the MNI MRI single-subject brain. Neuroimage 2002; 15:273-289.

36 Menon V, Uddin LQ: Saliency, switching, at tention and control: a network model of insula function. Brain Struct Funct 2010;214: 655-667.

37 Di Martino A, Scheres A, Margulies DS, Kelly AM, Uddin LQ, Shehzad Z, Biswal B, Walters JR, Castellanos FX, Milham MP: Functional connectivity of human striatum: a resting state FMRI study. Cereb Cortex 2008;18: 2735-2747.

38 Cservenka A, Casimo K, Fair DA, Nagel BJ: Resting state functional connectivity of the nucleus accumbens in youth with a family history of alcoholism. Psychiatry Res 2014;221: 210-219.

39 Naqvi NH, Gaznick N, Tranel D, Bechara A: The insula: a critical neural substrate for craving and drug seeking under conflict and risk. Ann N Y Acad Sci 2014; 1316:53-70.

40 Metcalf O, Pammer K: Physiological arousal deficits in addicted gamers differ based on preferred game genre. Eur Addict Res 2014; 20:23-32.

41 Rogers RD, Moeller FG, Swann AC, Clark L: Recent research on impulsivity in individuals with drug use and mental health disorders: implications for alcoholism. Alcohol Clin Exp Res 2010;34:1319-1333.
42 Sridharan D, Levitin DJ, Menon V: A critical role for the right fronto-insular cortex in switching between central-executive and default-mode networks. Proc Natl Acad Sci U S A 2008;105:12569-12574.

43 Farr OM, Hu S, Zhang S, Li CS: Decreased saliency processing as a neural measure of Barratt impulsivity in healthy adults. Neuroimage 2012;63:1070-1077.

44 Goto Y, Grace AA: Limbic and cortical information processing in the nucleus accumbens. Trends Neurosci 2008;31:552-558.

45 Mitchell DG: The nexus between decision making and emotion regulation: a review of convergent neurocognitive substrates. Behav Brain Res 2011;217:215-231.

46 Hanlon CA, Dowdle LT, Austelle CW, DeVries W, Mithoefer O, Badran BW, George MS: What goes up, can come down: novel brain stimulation paradigms may attenuate craving and craving-related neural circuitry in substance dependent individuals. Brain Res 2015;pii:S0006-8993(15)00188-2.

47 Weiland BJ, Welsh RC, Yau WY, Zucker RA, Zubieta JK, Heitzeg MM: Accumbens functional connectivity during reward mediates sensation-seeking and alcohol use in high-risk youth. Drug Alcohol Depend 2013;128:130139.

48 Schacht JP, Anton RF, Myrick H: Functional neuroimaging studies of alcohol cue reactivity: a quantitative meta-analysis and systematic review. Addict Biol 2013;18:121-133.

49 Ko CH, Liu GC, Yen JY, Chen CY, Yen CF, Chen CS: Brain correlates of craving for online gaming under cue exposure in subjects with Internet gaming addiction and in remitted subjects. Addict Biol 2013;18:559-569.

50 Menon V, Uddin LQ: Saliency, switching, attention and control: a network model of insula function. Brain Struct Funct 2010;214 655-667.

51 van den Heuvel OA, Van Gorsel HC, Veltman DJ, Van Der Werf YD: Impairment of executive performance after transcranial magnetic modulation of the left dorsal frontal-striatal circuit. Hum Brain Mapp 2013;34:347-355. 